\title{
Roll-strip Interfacial Heat Fluxes in Twin-roll Casting of Low- carbon Steels and Their Effects on Strip Microstructure
}

\author{
R. P. TAVARES, M. ISAC ${ }^{1)}$ and R. I. L. GUTHRIE ${ }^{2)}$ \\ Formerly McGill University. Now at Departamento de Engenharia Metalurgica, Federal University of Minas Gerais, Rua Esprito \\ Santo, 35-Segundo Andar, Belo Horizonte, 30160,030-MG, Brazil. 1) On leave from Polytechnic University of Bucharest, \\ Romania. Department of Mining and Metallurgical Engineering, McGill University, Montreal, H3A 2B2, Canada. \\ 2) Department of Mining and Metallurgical Engineering, McGill University, Montreal, H3A 2B2 Canada. McGill Metals
} Processing Centre, Canada.

(Received on May 11, 1998; accepted in fian/ form on August 10, 1998)

\begin{abstract}
In twin-roll casting, interfacial heat transfer between the rolls and the solidifying metal can affect strip quality and limit productivity.

In the present work, interfacial heat fluxes between the roll and the solidifying metal were evaluated in a pilot twin-roll caster used in the production of strips of low-carbon steel. This evaluation was based on an inverse heat transfer analysis and on readings of thermocouples inserted at different positions of the roll sleeve of the IMI pilot scale TRC caster in Boucherville, Quebec.

Variations in transient roll-melt interfacial heat fluxes during contact of the freezing shells with the roll surfaces exhibited two patterns, one characterized by a simple peak, the other by a double peak in the heat flux versus time curves, depending on the casting conditions. Mechanisms explaining the two types of curve are advanced. Solidification and as-cast structures of strips produced in the pilot caster were also studied, and their characteristics analyzed in terms of fluid flow and heat transfer within the caster's sump.
\end{abstract}

KEY WORDS: twin-roll casting; metal-mould heat transfer; solidification; as-cast structure.

\section{Introduction}

During twin-roll casting, the molten metal solidifies by losing heat through its interfaces with the rolls. The rate of heat extraction along a roll's interface with the molten metal can affect both the surface and sub-surface quality of the strip, depending on numerous attendant variables such as:

- material used for roll construction;

- surface texture of rolls and characteristics of the surface coatings applied;

- thermal expansion of the rolls;

- metallostatic pressure;

- formation of air gaps.

In a previous paper, the McGill authors, in collaboration with F. Hamel, of IMI, studied the kinetics of heat extraction from the steel within the sump of the caster, to the twin rolls.

In modeling heat flows from the cooling liquid and forming strip into the rolls, the heat transfer coefficient, $h$, has generally been assumed to be constant along the roll-strip interface. This average heat transfer coefficient is chosen so as to match observed surface temperatures of the strip exiting the roll bite and/or temperatures measured inside the rolls. ${ }^{1)}$ Another approach commonly adopted involves choosing a value for $h$ that generates cooling rates compatible with the secondary dendrite arm spacings measured from the strip's microstructure. ${ }^{2)}$ Recently, it has also been suggested that two values of heat transfer coefficients could better reproduce this heat exchange process. ${ }^{2)}$

However, it is unlikely that the transient variations in heat exchange rates during roll contact with the metal could be accurately reproduced by either one, or even two, heat transfer coefficients. Moreover, the estimate of these coefficients, based on surface temperatures of the strip measured after the roll nip or on secondary dendrite arm spacings, is something of a trial-and-error procedure, prone to significant amounts of uncertainty. A continuous or semi-continuous evaluation of the heat fluxes between the rolls and the solidifying strip, based on sensors installed close to the rolls surfaces, is evidentally far more appropriate, but technically a more challenging.

In conventional continuous casting operations for slabs, blooms and billets, the as-cast microstructure is completely, or almost completely, changed during reheating operations. In strip casting processes, the ascast structure is of major significance when the reheating stage is eliminated and direct rolling applied. This structure should be affected by the heat fluxes at the roll-strip interface. Little has been published regarding the as-cast structure of low-carbon steels strips produced by twin-roll casting. The investigation of Shiang and 
Wray ${ }^{3)}$ was mainly devoted to an analysis of how strip microstructures respond to different thermal processing operations. Ueshima et al. ${ }^{4)}$ studied the effect of $\mathrm{MnS}$ precipitation during the strip's solidification, on ferrite grain size distribution. None of these works correlated the characteristics of the microstructures, particularly grain sizes, to the corresponding rates of heat extraction and to caster conditions.

In the present work, ${ }^{6)}$ an inverse heat transfer analysis was performed and readings of thermocouples inserted in one of the rolls of a pilot twin-roll caster were used to determine semi-continuous variations in heat fluxes and heat transfer coefficients between the roll and the strip for two different casting conditions. The solidification structures of low-carbon steel strips produced in the same pilot caster were also studied and characterised in terms of associated casting conditions and measured rates of heat extraction.

\section{Methodology}

The investigation was divided in two parts:

- evaluation of roll-strip interfacial heat fluxes by an inverse heat transfer analysis;

- characterization of solidification and as-cast structures of the strips produced in the pilot caster.

\subsection{Evaluation of Roll-strip Interfacial Heat Fluxes}

\subsubsection{Experimental Set-up and Procedures}

Heat fluxes at one of the roll-strip interfaces of a pilot twin-roll caster were deduced from embedded thermocouple data. The main characteristics of this caster and casting conditions used during these experiments are given in Table 1. In all experiments, the roll separating force was kept approximately constant at $20 \mathrm{kN}$ or $0.2 \mathrm{~atm} \mathrm{~m}^{2}$. Three low-carbon steels were cast. Their chemical analyses are given in Table 2 .

Three pairs of Chromega-Alumega*) (type K) ungrounded thermocouples, $0.25 \mathrm{~mm}$ in diameter, were inserted through $0.5 \mathrm{~mm}$ diameter holes drilled in the copper-alloy sleeve of one of the rolls. Figure 1 provides a schematic representation of the experimental set-up in the pilot caster.

Each pair of thermocouples was located at a different position along the roll width. They were all inserted in one half of a roll, at $3,6.5$ and $10 \mathrm{~cm}$ away from the edge of the roll in contact with the side dam. The exact positions of the thermocouples inside the roll sleeve were determined using a laser ultrasonic inspection system, whose resolution was estimated to be $\pm 0.1 \mathrm{~mm}$. These positions are given in Table 3. Temperature data was acquired at a frequency of 2 or $10 \mathrm{~Hz}$ during the entire casting trials, which corresponded to approximately $250 \mathrm{~s}$, or 9 to 14 turns of the rolls, depending on the casting speed.

Table 1. Characteristics of the twin roll caster and casting conditions.

\begin{tabular}{lc}
\hline \multicolumn{1}{c}{ Caster characteristics } & Value \\
\hline Roll diameter $(\mathrm{m})$ & 0.60 \\
Roll width $(\mathrm{m})$ & 0.20 \\
Thickness of roll sleeve $(\mathrm{m})$ & 0.020 \\
\hline Casting conditions & Value \\
\hline Casting speed (m/s) & $0.067-0.133$ \\
Strip thickness (m) & $4-7.5 \times 10^{-3}$ \\
Contact angle (degrees) & 40 \\
\hline
\end{tabular}

Table 2. Chemical analysis of low-carbon steels.

\begin{tabular}{lllllllll}
\hline & \multicolumn{7}{c}{ Element } \\
Steel & \multicolumn{1}{c}{$\mathrm{C}$} & $\mathrm{Mn}$ & $\mathrm{Si}$ & $\mathrm{P}$ & $\mathrm{S}$ & $\mathrm{Al}$ & $\mathrm{N}$ \\
& \multicolumn{1}{c}{$\mathrm{C}$} & & & & & & \\
\hline $\mathrm{A}$ & 0.11 & 0.42 & 0.087 & 0.009 & 0.009 & 0.0004 & 0.0056 \\
$\mathrm{~B}$ & 0.127 & 0.42 & 0.120 & 0.012 & 0.012 & 0.0030 & 0.0065 \\
$\mathrm{C}$ & 0.146 & 0.38 & 0.10 & 0.008 & 0.005 & 0.002 & 0.010 \\
\hline
\end{tabular}

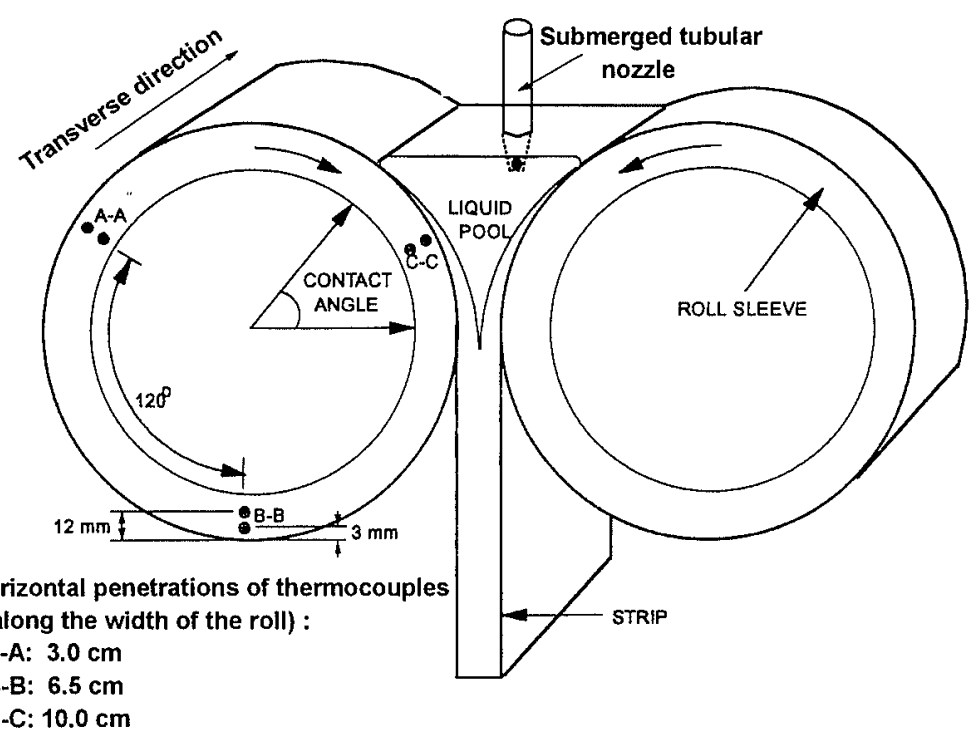

Fig. 1. Schematic representation of the experimental set-up in the pilot caster.

\footnotetext{
*) Chromega-Alumega is a trademark of Omega Inc.
} 
Table 3. Thermocouple locations within roll sleeve.

\begin{tabular}{cc|c}
\hline \multicolumn{2}{c|}{ Thermocouples } & $\begin{array}{r}\text { Distance to the } \\
\text { external surface } \\
(\mathrm{mm})\end{array}$ \\
\hline $\begin{array}{c}\text { Pair A-A } \\
30 \mathrm{~mm} \text { to the side }\end{array}$ & $\begin{array}{c}\text { External } \\
\text { Internal }\end{array}$ & $\begin{array}{r}4.5 \\
11.6\end{array}$ \\
\hline Pair B-B & External & 3.2 \\
$65 \mathrm{~mm}$ to the side & Internal & 12.7 \\
\hline Pair C-C & External & 3.0 \\
$100 \mathrm{~mm}$ to the side & Internal & 12.0 \\
\hline
\end{tabular}

\subsubsection{Inverse Heat Transfer Analysis}

The temperatures measured in the roll sleeve were then used in the solution of an inverse heat transfer problem to determine the interfacial heat fluxes. When thermocouples are used in the evaluation of transient heat fluxes by means of inverse methodologies, the response time of the thermocouple may corrupt the temperature history, affecting both the magnitude and timing of heat flux variations, especially if, as the present work illustrates, the heat fluxes change rapidly. ${ }^{5)}$ In such situations, it is important to characterize the responses of the thermocouples so as to make any necessary adjustments in the temperature data for any estimate of the heat fluxes to be accurate.

Since it was not possible to determine the response time in situ, this measurement had to be performed using an apparatus that reproduced the conditions found in the pilot caster. The apparatus and technique used in the correction for temperatures are described in the work of Tavares. ${ }^{6)}$

In evaluating interfacial heat fluxes, a Lagrangian coordinate system, moving with the same speed of the rolls and thermocouples, was used. In this system, the heat transfer inside the roll can be expressed in terms of Fourier's heat conduction equation. Heat conduction in the angular and axial directions of the roll was neglected. Based on these assumptions, the heat conduction equation is reduced to the following one-dimensional form:

$$
\rho C_{\mathrm{p}} \frac{\partial T}{\partial t}=k\left[\frac{1}{r} \frac{\partial}{\partial r}\left(r \frac{\partial T}{\partial r}\right)\right]
$$

where: $\rho=$ density;

$C_{\mathrm{p}}=$ specific heat;

$k=$ thermal conductivity;

$T=$ temperature;

$t=$ time;

$r=$ radial position.

A second order regularization method ${ }^{7)}$ was used in the solution of the inverse heat transfer problem. Since a pair of thermocouples located at two radial positions were used for each of the three distances to the side of the roll, Eq. (1) was subjected to the following initial and boundary conditions:
Table 4. Data used in the solution of the inverse heat conduction problem.

\begin{tabular}{cc}
\hline \multicolumn{1}{c}{ Data } & Value \\
\hline Roll sleeve $($ copper alloy) & \\
- density $\left(\mathrm{kg} / \mathrm{m}^{3}\right)$ & 8820.0 \\
- specific heat $(\mathrm{J} / \mathrm{kg} \mathrm{K})$ & 410.03 \\
- thermal conductivity $(\mathrm{W} / \mathrm{m} \mathrm{K})$ & 367.26 \\
- thermal diffusivity $\left(\mathrm{m}^{2} / \mathrm{s}\right)$ & $1.02 \times 10^{-4}$ \\
Coating & \\
- thermal diffusivity ${ }^{9)}\left(\mathrm{m}^{2} / \mathrm{s}\right)$ & $1.26 \times 10^{-5}$ \\
- thickness $(\mathrm{m})$ & $1 \times 10^{-4}$ \\
\hline
\end{tabular}

$\begin{array}{lrlr}\text { I.C. } & \text { (i) } & t=0: T=T_{\mathrm{i}}(r) & R_{\mathrm{i}} \leq r \leq R \\ \text { B.C.1 } & \text { (ii) } & t>0: r=R_{\mathrm{i}} & T=T_{\mathrm{TC} 1}(t) \\ \text { B.C.2 } & \text { (iii) } & t>0: r=R & -k \frac{\partial T}{\partial r}=q_{\mathrm{R}}(t)\end{array}$

where: $T_{\mathrm{i}}=$ initial temperature profile in the roll's sleeve;

$R_{\mathrm{i}}=$ radial position of the internal thermocouple, closest to the water cooling channels;

$T_{\mathrm{TC} 1}=$ temperature measured by the internal thermocouple (after correction);

$R=$ external radius of the roll sleeve; $q_{\mathrm{R}}(t)=$ heat flux at the roll-steel melt interface.

The temperatures measured by the internal thermocouples located further from the roll surface were used as boundary conditions while those determined by the external thermocouples closest to the interface were used as the fitting parameter for evaluating transient interfacial heat fluxes, $q_{\mathrm{R}}$. The heat transfer equation was solved for the region between the position of the internal thermocouple and the external surface of the roll.

Table 4 shows the data used for solving the inverse heat conduction problem. It includes the physical properties of the roll and details of the coating that was applied to its surface.

The one-dimensional approximation proposed for the present work was confirmed through numerical simulations in which transient local temperatures inside the roll sleeve were predicted by the solution of a three dimensional heat transfer model using known heat fluxes and roll speeds. These temperatures were then used in the one-dimensional algorithm above to back-calculate the heat fluxes applied. These fluxes were in excellent agreement with those used in the temperature generation. ${ }^{6)}$ This confirmed that circumferential and axial conduction of heat were negligible compared to radial heat conduction, for the range of roll speeds and roll material used in the present investigation.

\subsection{Solidification and As-cast Structures of the Strips}

Samples of the strips produced in the pilot caster were cut and various transverse sections across their width were analyzed. Samples for metallographic observation were prepared in the normal manner. Two different reagents were used in etching: $2 \%$ nital to reveal the as-cast structures, Oberhoffer's reagent to reveal the solidification or dendritic structure. 


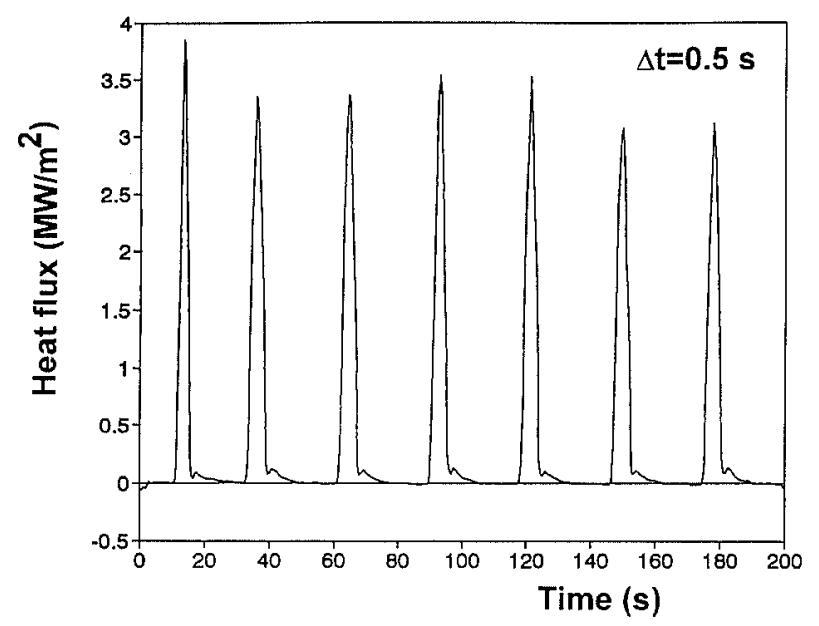

Fig. 2. Transient variations in heat fluxes at the roll-melt interface, during a group 1 experiment. Thermocouples: pair B-B.

\section{Results and Discussion}

\subsection{Roll-strip Interfacial Heat Fluxes}

The experiments were grouped according to the casting speed and thickness of the strips produced. Two groups were identified :

- Group 1: "low" casting speed, $0.067 \mathrm{~m} / \mathrm{s}(4 \mathrm{~m} / \mathrm{min})$, and thick strips, 6 to $7.5 \mathrm{~mm}$. Steels $A$ and $B$ of Table 3.2 were cast under these conditions;

- Group 2: "high" casting speed, 0.12 to $0.13 \mathrm{~m} / \mathrm{s}$ (7 to $8 \mathrm{~m} / \mathrm{min}$ ) and thin strips, $4 \mathrm{~mm}$. Steel $\mathrm{C}$ was cast in this group of experiments.

A tubular submerged entry nozzle (SEN), with two horizontal ports in the direction of the side dams, was used to deliver liquid steel into the caster. In all the runs, the superheat was approximately $25^{\circ} \mathrm{C}$.

Figure 2 shows a typical variation of the heat fluxes during one of the experiments in group 1. The peaks in the heat fluxes correspond to those periods of contact between that part of the roll containing a set of embedded thermocouples and the solidifying metal. The value of heat flux at the peak changes from one roll revolution to another. This is probably due to small variations in the casting conditions and also to the fact that, at each turn, the temperatures are sampled at different locations along the contact region. Since the heat fluxes change continuously during contact, different positions for sampling temperatures, naturally lead to different heat fluxes.

Figure 3 reveals in more detail, variations in heat fluxes during contact. After the beginning of metal contact with an entering segment of roll, there is a sharp increase in the heat flux. A maximum is reached, usually after $1 / 2$ to $2 / 3$ of the contact time.

This type of behaviour in local transient heat fluxes during solidification has been observed in other investigations, ${ }^{10-14)}$ and different mechanisms have been proposed.

In the case of the pilot twin-roll caster, the variation in heat flux with time of contact derives from an interplay of several factors, such as; the presence of an entrapped

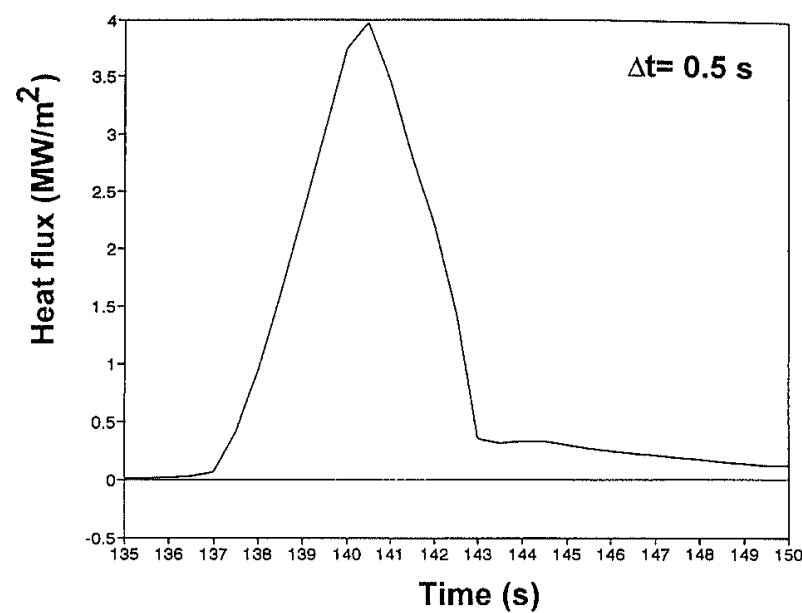

Fig. 3. Transient variations of heat fluxes during the contact time. Group 1 experiment. Thermocouples: pair $\mathrm{A}-\mathrm{A}$.

air film between the roll and the thin solidified shell, the thermal expansion of the rolls, the metallostatic pressure and solidification shrinkage. The following mechanism is proposed to account for the observed variation in heat fluxes:

(i) liquid steel is injected through the submerged entry nozzle and loses most of its superheat before reaching the meniscus region. As a consequence, solidification starts at the moment of contact of this metal with the cold roll surface;

(ii) heat transfer occurs between the surface of the roll and the surface of a very thin solidified shell. These two surfaces are rough (at macro- and microscopic scales), such that in certain points they are in contact while in other regions, they are separated by an entrapped film of air ${ }^{15)}$ or even carbon monoxide, for steel chemistries approaching rimming grade conditions. ${ }^{30)} \mathrm{At}$ the contact points, heat is transferred by conduction between the two solid surfaces. In the non-contact regions, conduction and radiation through the air film are the main mechanisms for heat transfer;

(iii) the initial contact between the two surfaces is not very adherent, which leads to a relatively small heat flux;

(iv) as the contact progresses, the roll expands due to a rise of its temperatures. ${ }^{16)}$ At the same time, the metallostatic pressure over the thin solidified shell increases, as a result of the increasing height of liquid metal above it. Consequently, the contact pressure tends to augment and the thickness of the air film to diminish. Both factors lead to higher interfacial heat fluxes;

(v) as heat is extracted from the metal, the solidified shell becomes thicker and strong enough to support the metallostatic pressure. Solidification shrinkage also becomes significant. These two factors cause a decrease in the heat flux.

It seems that, before reaching the maximum heat flux, the factors mentioned in item (iv) are more important and determine the variation in the heat flux. After the maximum, the effects listed in item (v) presumably take over and are responsible for the observed decrease in the heat fluxes. Independent evidence to support the present observations and proposed mechanism can be found in 


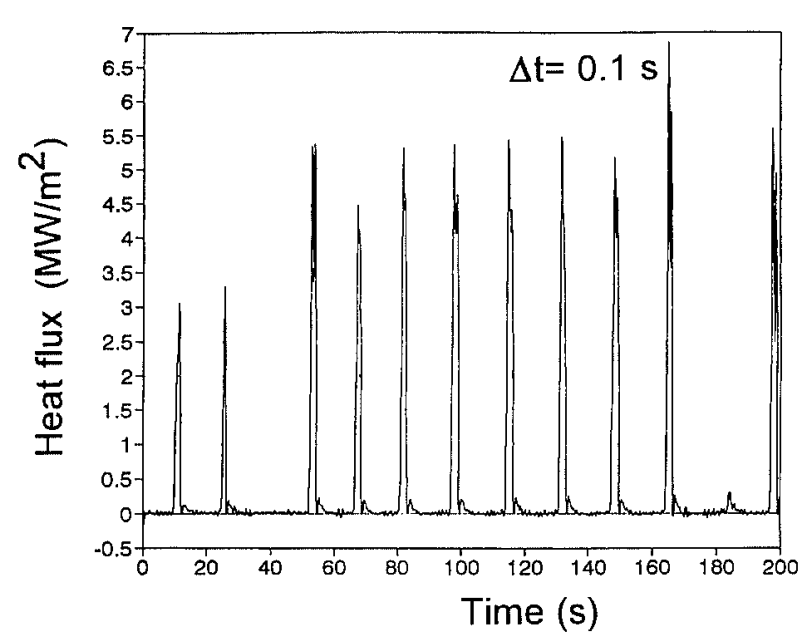

Fig. 4. Transient variations of heat fluxes at the roll-melt interface, during a group 2 experiment. Thermocouples: pair B-B.

the investigations of Nishida et al., ${ }^{12)}$ Mizoguchi et al. ${ }^{15)}$ and Rapier et al. ${ }^{17)}$

Figure 4 illustrates the variation in heat fluxes during one of the experiments in the group 2 series. In general, the peak heat fluxes were higher than those obtained in the group 1 experiments. This is probably due to the different casting speeds and also to the different compositions of steel being cast. The variation in heat flux during one of the periods of contact is presented in Fig. 5. Up until approximately $2 / 3$ of the contact time, the heat flux variation is similar to that shown in Fig. 3; a sharp increase until a maximum is reached, followed by a period of decrease. Most likely, the same mechanism proposed for the experiments of group 1 also applies here. The difference appears in the last $1 / 3$ of the contact time. For most of the experiments, a second peak in the heat flux occurs in this final period of contact.

The second peak in the heat flux is probably associated with a more intense interaction of the dendritic mushy zones formed around each roll as the solid fraction at the center of the caster continuously increases. At some critical solid fraction, the dendrites form a cohesive network that begins to develop some strength ${ }^{18)}$ and to exert pressure against the thin solidified shell, as the distance between the surfaces of the rolls diminishes. The thin solid shell is not able to stand this pressure and is pushed against the surface of the roll, thereby increasing the contact pressure and the heat flux between them. Similar to equivalent phenomena in rolling, the contact pressure reduces after reaching a maximum, ${ }^{19)}$ which explains the decrease in the heat flux close to the roll nip (end of contact). This "rolling" of a partially, or eventually totally, solidified material would then be responsible for the second peak in the heat flux. Since one drive wheel of the caster was fixed, while the other floated under an approximately constant load of $20 \mathrm{kN}$, any corroboratory pressure changes could not be sensed.

The question begged is why this second peak did not occur in the group 1 experiments. One possible reason for this is the fact that the cooling rate during solidification at lower roll speeds and thicker strips is smaller and associated heat fluxes to the rolls lower. In

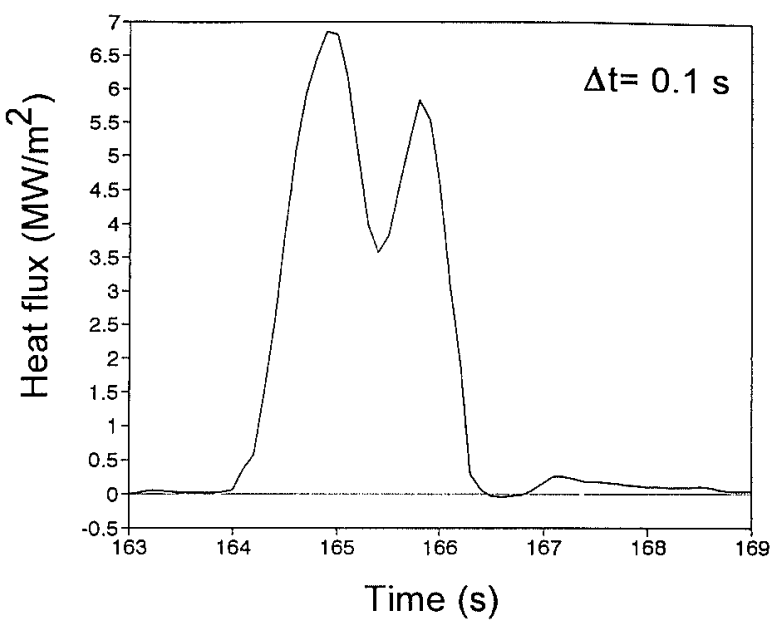

Fig. 5. Transient heat fluxes during one revolution of instrumented roll for a group 2 experiment. Thermocouples: pair $\mathrm{B} \cdots \mathrm{B}$.

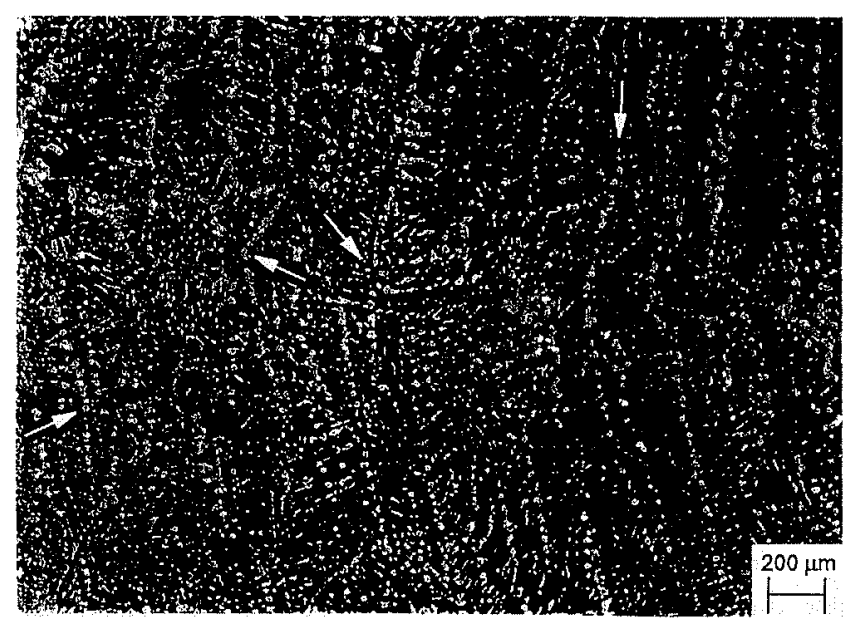

Fig. 6. Typical dendritic structure in the transverse section of one strip produced in a group 1 experiment.

this case, the dendrite arm spacings are larger and, as pointed out by Flemings, ${ }^{18)}$ the development of strength by the dendritic network would occur at a higher solid fraction, perhaps after the strip had already left the caster.

Although none of the group 1 experiments exhibited a second peak, there was in two cases a noticeable change in the slope of the heat flux curve that was observed close to the exit of the rolls. This might have been a sign of an incipient second peak. It should also be mentioned that, in these two cases, the strip being produced was thinner than the average for this group.

\subsection{Solidification and As-cast Structures of the Strips}

Samples from the two groups of casting conditions described in the previous sections were studied. The solidification or dendritic structure was revealed by Oberhoffer's reagent. Basically, this reagent reveals the different levels of microsegregation. The areas richer in iron (dendrites) appear black, while regions with higher concentrations of impurities (interdendritic spaces) appear white.

Figure 6 shows a transverse section of the dendritic structure of a strip produced in a group 1 experiment (low casting speed, thick strips). This figure gives more 
detail of the central region across the strip thickness. The structure is essentially columnar, with the columnar dendrites meeting approximately at the center of the strip. No evidence of an equiaxed region can be detected. However, several signs of interaction of the columnar dendrites coming from the two surfaces of the strip are observed. As indicated by the arrows, various broken and bent dendrites are seen. On the right side of the figure, one columnar dendrite seems to cross the center of the strip. Apparently, its growth was not blocked by other dendrites coming from the opposite surface. This is more likely to occur in situations of relatively large interdendritic spaces, associated with low cooling rates, which was exactly the case for the group 1 experiments.

The transverse section of the dendritic structure of a strip produced in one experiment of group two (high casting speed and thin strips) is illustrated in Fig. 7. Again, columnar dendrites are formed close to the surfaces of the strip. The primary dendrite spacings here are much finer than those presented in Fig. 6. This is in agreement with the higher cooling rates (higher heat fluxes) for the group 2 experiments. It is also seen that, opposed to what is shown in Fig. 6, the columnar dendrites do not reach the center of the strip. A zone with different characteristics appears in this region.

Previous investigations ${ }^{20,21)}$ have detected an equiaxed zone in the center of twin-roll cast strips. The forma- tion of this zone has been attributed to incomplete solidification of the strip at the roll gap. In the present study, it seems that another factor is responsible for the formation of this non-columnar zone. Simulations of heat transfer and solidification, ${ }^{6)}$ using the heat fluxes determined above, have shown that, in the experiments of group two, the strips are usually fully solid just above the roll gap. It is also seen in Fig. 7 that the non-columnar zone is darker than the surrounding areas. Considering

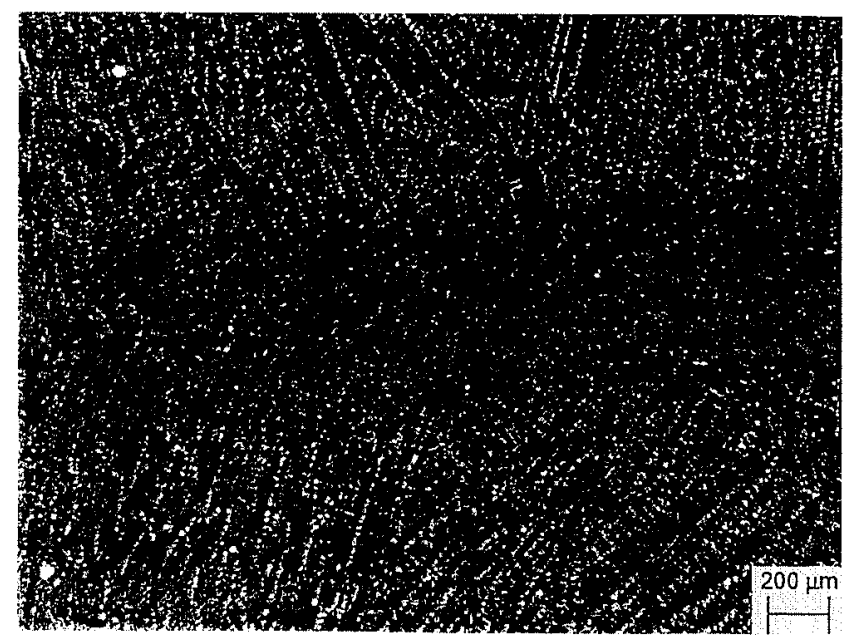

Fig. 7. Typical dendritic structure in the transverse section of one strip produced in a group 2 experiment.
Centre

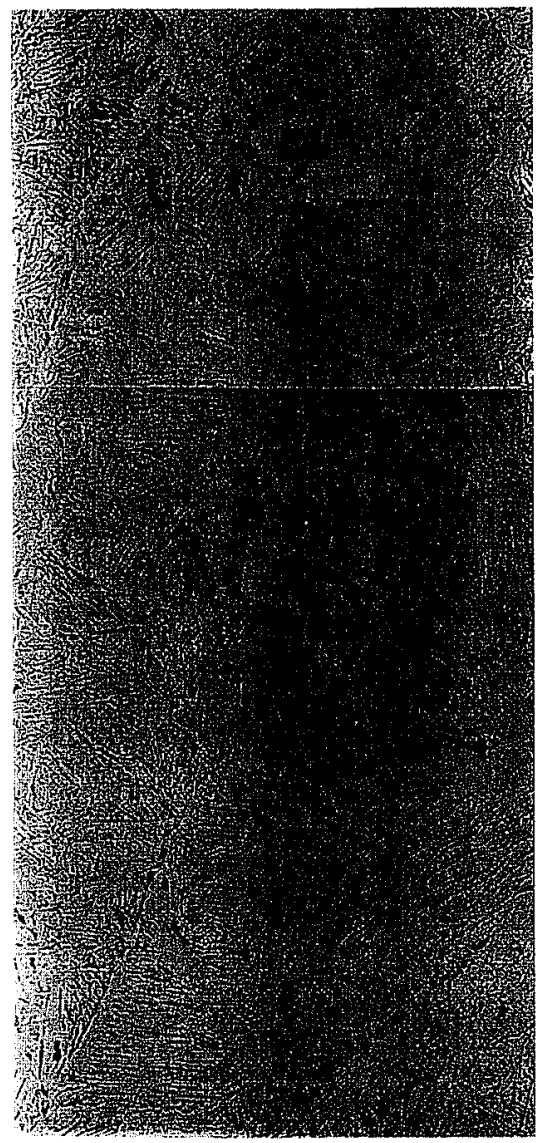

Surface
Centre

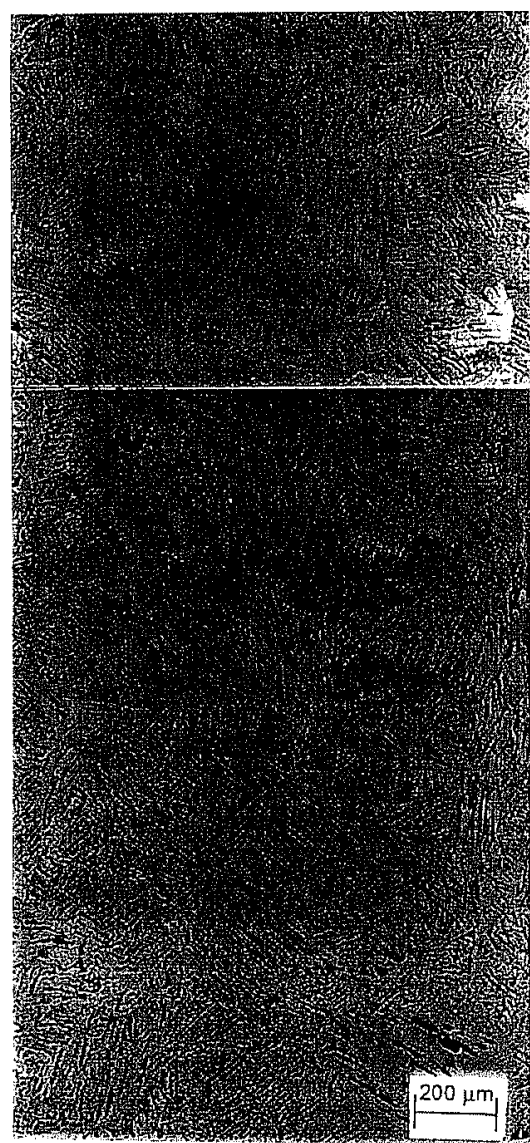

Surface

Fig. 8. Transverse section of the as-cast structure of a strip produced in a group 1 experiment. Section between the center and the edge of the strip. 
that the Oberhoffer's reagent makes the regions richer in impurities appear white, this might indicate that the non-columnar zone was depleted of these impurities, possibly due to squeezing of the interdendritic liquid back into the sump. This evidence suggests that a more intense interaction of dendrites and, a certain extent of hot deformation of the strip before leaving the rolls may have led to the formation of the non-columnar zone.

Figure 8 shows the as-cast structure across the thickness of one strip produced in a group 1 experiment. This section was taken from a region located approximately halfway between the center and the edge of the strip (along its width).

A hypoeutectoid steel was cast in this experiment and, as expected, the room temperature structure was mainly composed of ferrite and pearlite. The morphology of the ferrite in this sample is usually called Widmanstätten side plates. ${ }^{22,23)}$ This kind of morphology usually occurs when the prior austenite grains are large and the cooling rate during transformation is relatively high.

The Widmanstätten plates nucleate at the austenite grain boundaries and grow along well-defined matrix planes. ${ }^{23)}$ They grow either from the grain boundaries or from pre-existing ferrite allotriomorphs. Nucleation can also occur inside the grains, at the austenite twin planes. This kind of nucleation pattern, mainly at the grain boundaries, allows one to approximately identify the sizes and shapes of austenite grains prior to their transformation. They are delineated by the white network of ferrite. This feature was used to estimate the prior austenite grain sizes.

In Fig. 8, it is observed that the prior austenite grains are large, especially near the surface, where they show a tendency to have columnar shapes. A few approximate measurements of austenite grain sizes were made and the following average values were obtained:

- major length: $780 \mu \mathrm{m}$ (standard deviation: $328 \mu \mathrm{m}$, $n=10$ );

- minor length: $453 \mu \mathrm{m}$ (standard deviation: $117 \mu \mathrm{m}$, $n=10$ ).

These results are mainly for the surface region. In the center of the strip, the austenite grains that were identified present a more equiaxed shape, with sizes ranging from 130 to $360 \mu \mathrm{m}$. This variation in grain size across the strip's thickness can be analyzed in terms of previous works ${ }^{24-26)}$ that demonstrate that the austenite grains only grow in the temperature range where austenite is the single phase present. The surface of the strip reaches this range before the center and, consequently, the grains there have more time to grow. This certainly limits the grain growth in the central part, once it reaches the single phase temperature range. Simulations of heat transfer and solidification across the strip thickness ${ }^{6)}$ also indicate that the surface region experiences a significant reheating after leaving the roll gap. This reheating favours a more pronounced grain growth in surface regions of the strip.

The as-cast structure for one section in the mid-width of the strip is depicted in Fig. 9. Close to the surface, the structure is similar to that shown in Fig. 8. However, in the central region, it is significantly different, with smaller and more equiaxed ferrite grains. As pointed out
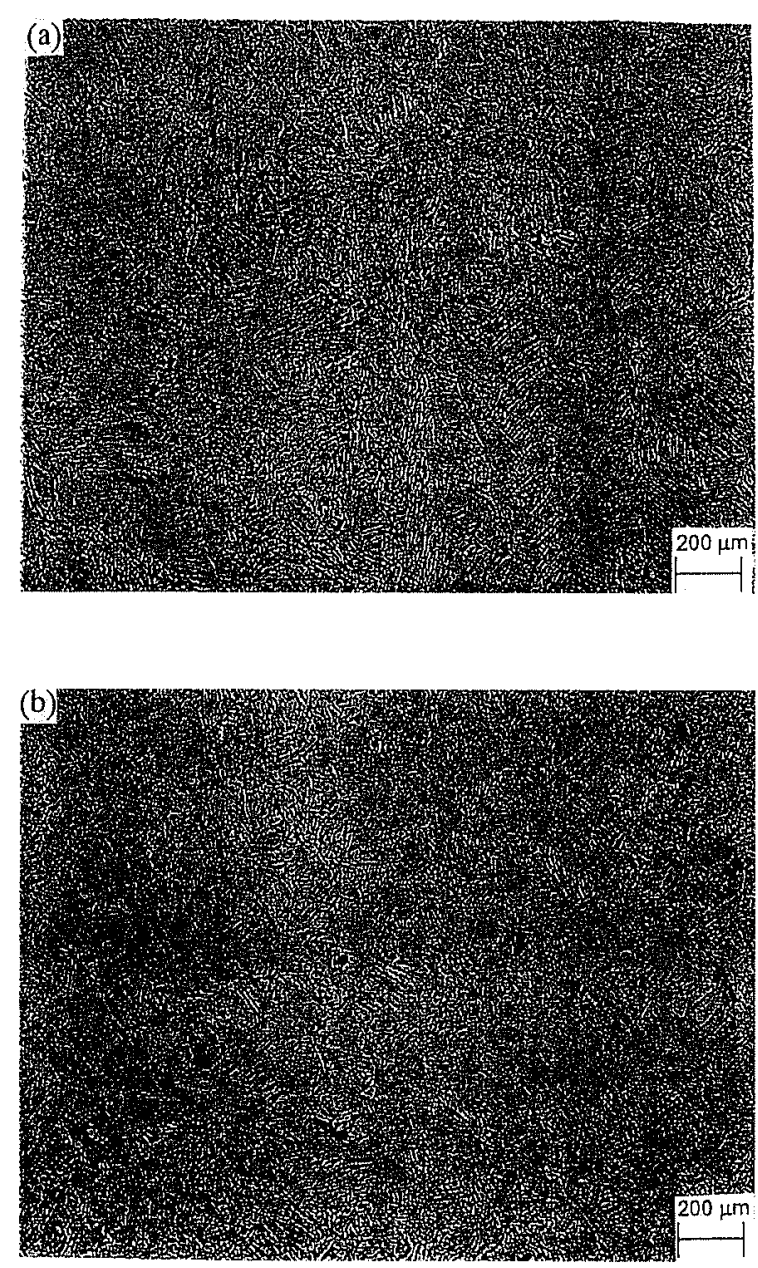

Fig. 9. As-cast structure in the mid-width of a strip produced in a group I experiment.

(a) Surface region (b) Central region

by Shewmon, ${ }^{22)}$ this kind of ferrite grain certainly originated from austenite grains that were much finer than those closer to the strip's two surfaces. The question is what caused the formation of this fine austenite, in this particular section of the strip. In the section presented in Fig. 8, the austenite is finer in the center, but apparently not sufficiently so, as to lead to the formation of equiaxed ferrite. A possible reason for this difference is now discussed.

Thus, in some recent work, Yasuda et al. ${ }^{27)}$ investigated the effects of deformation in the semi-solid state on the solidification structure of carbon steels and showed that reductions in the semi-solid state lead to grain refinement. For a given average solid fraction, more intense reductions cause more significant grain refinement.

A model for fluid flow, heat transfer and solidification in twin-roll casters ${ }^{28)}$ has predicted that the bifurcated SEN nozzle used in the pilot caster tends to give an unevenly solidified shell, with faster advance of the solidification fronts in the central regions of the converging steel shells. The combination of these results together with the information provided by the work of Yasuda et $a{ }^{2}{ }^{27)}$ may explain the different structures shown in Figs. 8 and 9. The more pronounced solidification in the center of the rolls causes the two solid- 


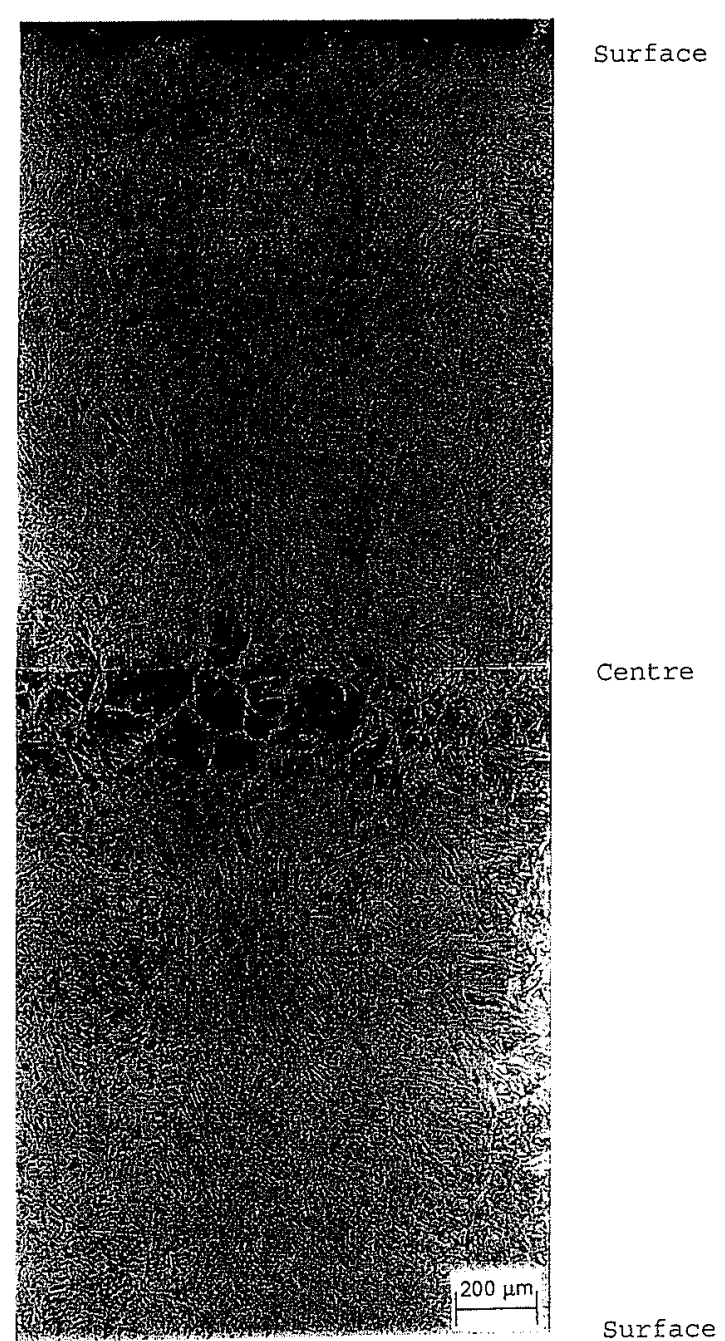

Fig. 10. Transverse section of the as-cast structure of a strip produced in a group 2 experiment. Section close to the edge of the strip.

ification fronts in this region to meet ahead of other locations along the widths of the rolls. Consequently, the partially solidified layer formed at the centers of the roll surfaces are subjected to more intense reduction before leaving the caster. As such, these grains would therefore exhibit a more significant degree of grain refinement. Furthermore, this grain refinement is more noticeable in the central part of the strip thickness because the strain tends to concentrate there, due to the higher temperature.

Figure 10 presents the as-cast structure across the thickness of one strip produced in an experiment of group two. This section was located close to the edge of the strip.

The structure seen in Fig. 10 is analogous to that shown in Fig. 8. In this sample, it is more difficult to identify the prior austenite grains. The few measurements that were made close to the surface gave the following results:

- major length: $205 \mu \mathrm{m}$ (standard deviation: $36 \mu \mathrm{m}$, $n=5$ );

- minor length: $168 \mu \mathrm{m}$ (standard deviation: $27 \mu \mathrm{m}$, $n=5$ ).

The higher cooling rates in the group 2 experiments (higher heat fluxes), as compared to those of group one, associated with smaller thicknesses of the strips, are certainly responsible for these smaller austenite grains. In the center of this sample, the equiaxed grain sizes are approximately $120 \mu \mathrm{m}$.

The dark area seen in the center of Fig. 10 indicates a more significant fraction of pearlite within the area. This may be caused by carbon segregation during solidification or also by the carbon rejected during the precipitation of ferrite from the austenite. In this case, the carbon rejection becomes more apparent due to the relatively small austenite grain size.

Similar to the group 1 samples, the as-cast structure in the mid-width of the group 2 strip also showed, in its central part across the thickness, a region with smaller and more equiaxed ferrite grains.

These results demonstrate that, within the range of strip thickness and casting conditions investigated, there is a tendency for large austenite grains to form following solidification, especially close to the strip's surfaces. This is particularly true for low casting speeds and thick strips. The size of these austenite grains is similar to those obtained after reheating of slabs for hot rolling. ${ }^{29)}$ However, in the case of slabs, there is a great amount of deformation to be applied before reaching the final thickness. The successive steps of deformation cause a significant reduction in the austenite grain size, so that when the slabs are reduced to the thicknesses of the strips produced in the pilot caster $(4-7 \mathrm{~mm})$, they present austenite grains which are much finer $(20-50 \mu \mathrm{m})$ than those observed in the twin-roll cast strips. Therefore, if the cast strips are to be hot rolled without cooling to ambient temperature and subsequent reheating, it seems important to reduce the austenite grain growth during the casting operation and to adjust the post-processing route to obtain products with mechanical properties within specifications. Whether this is possible for low carbon steels cast with twin rolls remain an open question.

\section{Conclusions}

Interfacial heat fluxes between the roll and the solidifying metal have been evaluated in a pilot twin-roll caster producing strips of low-carbon steel. This evaluation was based on an inverse heat transfer analysis and on readings of thermocouples inserted at different positions of the roll sleeve.

Transient variations in the roll-melt interfacial heat fluxes exhibited two different patterns, according to casting conditions:

- single peak : usually at low casting speeds and when thicker strips are produced;

- double peak : predominantly at higher casting speeds and for thinner strips.

The average heat flux in the case of the double peak pattern was higher than that of the single peak.

Mechanisms to explain these two types of behavior have been proposed, involving interfacial gas entrainment, ferrostatic pressure, shell shrinkage and substrate properties.

A study of the microstructure of the strips showed that the prior austenite grains, identified by nucleation of 
ferrite along the grain boundaries, exhibited average grain sizes that were well above those of conventionally processed materials ( $\sim 400 \mu \mathrm{m}$ vs. $20-50 \mu \mathrm{m}$ ), for the same product thickness.

The thicker strips, produced at low casting speeds, formed larger austenite grains. In this case, the interfacial heat fluxes were smaller (average values of $3 \mathrm{MW} / \mathrm{m}^{2} v s$. $4.5 \mathrm{MW} / \mathrm{m}^{2}$, for higher casting speed and thinner strips) since the strips remained at higher temperatures for longer periods. This time lapse favored significant $\gamma$ grain growth.

The presence of small equiaxed grains in the center of the strip thickness, particularly for thin strips, is a consequence of melt recirculation and reduction in the semi-solid state, due to the interaction of the mushy zones forming on the surface of the rolls.

\section{Acknowledgements}

The authors gratefully acknowledge the support of the Natural Science and Engineering Research Council of Canada, and a Research Scholarship from the Brazilian Government, for making this work possible. Similarly, we thank Prof. Dagoberto B. Santos of Federal University of Minas Gerais, Brazil, and colleagues at IMI Boucherville, for providing some of the various strip samples analysed.

\section{REFERENCES}

1) H. Yasunaka, K. Taniguchi, M. Kokita, S. Koyama and C. Yoshida: Proc. of the Int. Conf. on New Smelting Reduction and Near-Net-Shape Casting Technologies for Steels, Pohang, Korea, (1990), 570.

2) W. Löser, S. Thiem and M. Jurisch: Mater. Sci. Eng., A173 (1993), 323.

3) L.-T. Shiang and P. J. Wray: Metall. Trans. A, 20A (1989), 1191.

4) Y. Ueshima, T. Sawai, T. Mizoguchi, K. Miyazawa and S. Mizoguchi: Proc. of the 6th Int. Iron and Steel Cong., (1990), 642.

5) K. A. Woodbury: Int. J. Heat Mass Transfer, 33 (1990), No. 12, 2641.

6) R. P. Tavares: Ph. D. Thesis, McGill University, (1997).

7) J. V. Beck, B. Blackwell and C. R. St. Clair, Jr.: Inverse heat conduction. Ill-posed problems, John Wiley \& Sons, New York, (1985), $308 \mathrm{p}$

8) K. Yamamoto: The results of F.E.M. analysis for IVACO's twin-roll (Bessemer Projet), Chuetsu Metal Works, Internal Report, IMI, Montreal, (1993).

9) J.-G. Legoux, S. Dallaire, D. Larouche and A. Guillet: Proc. of the Int. Symp. on Development and Application of Ceramics and New Metal Alloys, CIM, Toronto, (1993), 273.

10) C. A. Muojekwu, I. V. Samarasekera and J. K. Brimacombe: Metall. Mater. Trans. B, 26B (1995), 361.

11) J. S. J. Chen, R. C. Ren and A. A. Tseng: J. Mater, Process. Manuf. Sci., 3 (1995), 373.

12) Y. Nishida, W. Droste and S. Engler: Metall. Trans. B, 17B (1986), 833.

13) B. Farouk, D. Apelian and Y. G. Kim: Metall. Trans. B, 23B (1992), 477.

14) G.-X. Wang and E. F. Matthys: Trans. ASME-J. Heat Transfer, 118 (1996), 157.

15) T. Mizoguchi, K. Miyazawa and Y. Ueshima: Tetsu-to-Hagané, 80 (1994), No. 1, 36.

16) T. Yamauchi, T. Nakanori, M. Hasegawa, T. Yaburi and N. Ohnishi: Trans. Iron Steel Inst. Jpn., 28 (1988), No. 1, 23.

17) A. C. Rapier, T. M. Jones and J. E. Mcintosh: Int. J. Heat Mass Transfer, 6 (1963), 397.

18) M.C. Flemings: Metall. Trans. B, 22B (1991), 269

19) G. E. Dieter: Mechanical Metallurgy, McGraw Hill, New York, (1983), 751.

20) T. Mizoguchi and K. Miyazawa: ISIJ Int., 35 (1995), No. 6, 771.

21) T. Mizoguchi and K. Miyazawa: Adv. Mater. Process., 1 (1990), 93.

22) P. G. Shewmon: Transformations in metals, McGraw Hill Book Co., New York, (1969), 394.

23) R. W. K. Honeycombe and H. K. D. H. Bhadeshia: Steels. Microstructures and Properties, John Wiley \& Sons, New York, (1995), $324 \mathrm{p}$.

24) Y. Maehara, K. Yasumoto, Y. Sugitani and K. Gunji: Trans. Iron Steel Inst. Jpn., 25 (1985), 1045.

25) K. Yasumoto, T, Nagamichi, Y. Maehara and K. Gunji: Tetsu-10-Hagané, 73 (1987), 1738.

26) K. Matsuura, Y. Itoh and K. Matsubara: Tetsu-to-Hagané, 76 (1990), 714

27) K. Yasuda, M. Minagawa and H. Kajioka: Tetsu-to-Hagané, 80 (1994), No. 12, 896

28) R. P. Tavares and R. I. L. Guthrie: Proc. of the Int. Symp. on Computational Fluid Dynamics and Heat/Mass Transfer Modelling in the Metallurgical Industry, CIM, (1996), 203.

$29)$ I. Tamura, H. Sekine, T. Tanaka and C. Ouchi: Thermomechanical Processing of High Strength Low-alloy Steels, Butterworths, London, (1988), $248 \mathrm{p}$

30) K. Amada, Y. Ueshima, T. Mizoguchi, K. Miyazawa and S. Mizoguchi: CAMP-ISIJ, 3 (1990), 1684. 